\title{
The influence of breast density on the sensitivity and specificity of ultrasound and mammography in breast cancer diagnosis
}

\author{
Svjetlana Mujagić, Mensura Burina, Hanifa Fejzić
}

Department of Radiology and Nuclear Medicine, University Clinical Center Tuzla Tuzla, Bosnia and Herzegovina

Corresponding author: Svjetlana Mujagić

Ahmeta Kobića 19 75000 Tuzla

Bosnia and Herzegovina svjetlanabh@yahoo.com

Tel.: + 38761661080

$+38735394242$

Fax.: + 38735251456

Received: 16 June 2011

Accepted: 10 September 2011

Copyright (C) 2011 by Academy of Sciences and Arts of Bosnia and Herzegovina. E-mail for permission to publish: amabih@anubih.ba
Objective. The aim of this study was to analyse the sensitivity and specificity of ultrasound and mammography according to breast density and determine which of these diagnostic imagings is a more accurate test for diagnosis of breast cancer. Patients and methods. By means of a cross-sectional study, ultrasound and mammographic examinations of 148 women with breast disease symptoms were analysed. All women underwent surgery and all lesions were examined by histological examination which revealed the presence of 63 breast cancers, and 85 benign lesions. Histological examination was used as the "gold standard". In relation to breast density, the women were separated into two groups, group A: women with "fatty breast" (ACR BI-RADS density categories 1 and 2) and group B: women with "dense breast" (categories 3 and 4). Ultrasound and mammographic findings were classified on the BI-RADS categorical scale of 1-5. For statistical data processing, the logistic regression analysis and the McNemar chi-square test for paired proportions was used. The differences on the level of $p<0.05$ were considered statistically significant. Results. In the group of women with breast density categories 1 and 2 the difference in the sensitivities $(p=1)$ as well as in the specificities $(\mathrm{p}=0.11)$ of the two imaging tests was not statistically significant. In the group of women with breast density categories 3 and 4 the ultrasound sensitivity was significantly higher than the mammographic sensitivity $(\mathrm{p}=0.03)$ without a statistically significant difference in specificity $(\mathrm{p}=0.26)$. Sensitivity of mammography was (linearly - ex; linearity exists between breast density and the logarithm of odds for a positive result) associated with breast density (likelihood ratio $\chi^{2}=15.99, \mathrm{p}=0.0001$ ). The odds ratio for (the probability of ex) a positive mammographic result was 0.25 (95\% CI, 0.11-0.58). The sensitivity of ultrasound and specificity of each test were not (linearly - ex) associated with breast density. Conclusion. Breast density had a significant influence on the sensitivity of mammography but not on specificity. This is very important because a certain percentage of women, not only under 40 but also after 40 , have heterogenous and extremely dense breasts (density categories 3 and 4). In these women, ultrasound is a more accurate imaging test than mammography, while in the women with fatty breasts (density categories 1 and 2) these imaging tests are almost equally accurate in breast cancer diagnosis.

Key words: Breast cancer, Breast density, Ultrasound, Mammography. 


\section{Introduction}

Mammography and breast ultrasound are the most common diagnostic imagings used in breast cancer detection. Mammography is used as a screening method for early detection of breast cancer in women after 40 , and as diagnostic mammography in symptomatic women, when a breast lump or nipple discharge is found during self-examination or an abnormality is found during screening mammography $(1,2)$. Breast ultrasound is used to evaluate specific abnormalities discovered either on mammography or at clinical examination $(3,4)$.

Although the sensitivity of mammography in the diagnosis of breast cancer is influenced by age, family history, body mass index and some other factors, one of the most important is the inherent limitation of mammography in breast cancer diagnosis is breast density $(4,5)$. Breast density, which refers to the prevalence of fibroglandular tissue in the breast as it appears on a mammogram, is also associated with increased risk of breast cancer $(6,7)$. In recent years, the most commonly used method for evaluation and reporting breast density in mammography is the BI-RADS (Breast Imaging Reporting and Data System) proposed by The American College of Radiology (ACR). According to the ACR BI-RADS, breast density is graded on a scale of 1 to 4: (1) almost entirely fat breast; (2) scattered fibroglandular tissue; (3) heterogenous breast, (4) extremely dense breast (8). A global evaluation of breast density and classification leads to the possibility that breast cancer in the mammography image is not revealed. The success of mammography is limited in the group of women with density categories 3 and 4 , especially younger women $(2,5)$. In these two groups there are a number of false negative findings because heterogenous and extremely dense tissue appears white on the mammography image, as does breast can- cer ("white breast-white cancer") making it more difficult to distinguish between the two. In contrast, dense glandular tissue usually has a hyperechoic appearance on ultrasound. Because most breast cancers are hypoechogenic, carcinomas in this setting are easily detected on ultrasound ("white breastdark cancer") $(4,9)$.

The degree of breast density decreases with age, but a certain percentage of women in menopause have extremely dense breasts. Women who have not given birth or who have had only one child, those who have not breastfed, and women who use contraception or hormone replacement therapy have a higher degree of breast density (10).

The aim of this study was to analyse the sensitivity and specificity of ultrasound and mammography according to breast density and determine which of these two diagnostic imagings is a more accurate test for diagnosis of breast cancer.

\section{Patients and methods}

By means of a cross-sectional study, ultrasound and mammographic examinations were analysed of 148 women with breast disease symptoms or positive family history for breast cancer in the period from January 2009 to November 2010. All the women underwent surgery, and all 148 breast lesions were examined by histopathology analysis, which was used as the "gold standard". Histopathology results revealed the presence of 63 breast cancers, and 85 benign lesions. Diagnostic imaginings were performed at the Department of Radiology and Nuclear Medicine, surgical treatment at the Department of Surgery, and pathohistological analysis at the Department of Pathology of the Polyclinic for Laboratory Diagnostics of the University Clinical Centre, Tuzla. The group pattern was made consecutively.

We evaluated the density of breast parenchyma according to the gradation of the 
American College of Radiology BI-RADS

(10) protocol on a scale of 1-4:

1. almost entirely fat breast

2. scattered fibroglandular tissue

3. heterogenous breast

4. extremely dense breast

In relation to breast density, the women were separated into two groups, group A: women with "fatty breast" (categories 1 and 2 ) and group B: women with "dense breast" (categories 3 and 4).

The breast ultrasound was performed on a "Sonoline G60 S"- Siemens ultrasound machine with 7.5 MHz-linear array transducer and if needed with a $12 \mathrm{MHz}$-linear array transducer. Mammography was performed on a Siemens "Mammomat Nova 3000". Standard mediolateral oblique and craniocaudal views of each breast were taken. To obtain and read mammography images, cassettes with phosphorus imaging plates (18x24 and 24x30), mamma-laser drystar DT-2 films and digitizer type CR $85-\mathrm{X}$ with an NX workstation were used. The findings were interpreted by two radiologists. Additionally, according to the radiological features of the described pathological changes, ultrasound and mammographic findings were classified on the BI-RADS categorical scale of 1-5 (4) as follows:

1. no significant abnormality

2. benign finding

3. probably benign finding

4. suspicious lesions- suspicious abnormality

5. highly suggestive of malignancy -malignant lesion

Categories 1, 2 and 3 were considered negative, while categories 4 and 5 were considered as positive for cancer.

\section{Statistical analysis}

The standard methods of descriptive statistics (mean and standard deviation) and nonparametric $\mathrm{McNemar}$ chi-square test for paired proportions were used for statistical data processing. The sensitivity and specificity of the methods were determined by the $2 \times 2$ table diagnostic test. The differences on the level of $\mathrm{p}<0.05$ were considered statistically significant. Logistic regression analysis was performed using statistical software MedCalc (version 11.4.2.0). The logarithm of the odds of a positive result was regressed on breast density for cancer patients for each imaging modality to assess the influence of breast density on test sensitivity. The logarithm odds of a negative result were regressed on breast density for subjects without cancer for each imaging modality to assess the influence of breast density on test specificity.

\section{Results}

The study included 148 patients, 63 patients with breast cancer and 85 patients with benign lesions determined by the histopathology examination. The mean age of all the patients was $51.6 \pm 10.8$ years, ranging from 19 to 79 years. The mean age of patients with breast cancer was $55.4 \pm 11.4$, while the mean age of patients with benign lesions was $48.8 \pm 9.4$. In the study $36(24.3 \%)$ women were included with almost entirely fat breast (categories 1), 40 (27\%) women with scattered fibroglandular tissue (categories 2), 56 (37.8\%) women with heterogenous breasts (categories 3), while 16 (10.8\%) women had extremely dense breasts (categories 4). Figure 1 shows distribution density categories in different age groups. Dense breasts were found in $87.5 \%$ women under 40 years; $70.7 \%$ women aged $41-50 ; 25.5 \%$ women aged $51-60$ and $18.5 \%$ women above 60 years (Figure 1).

In group A (women with fatty breastdensity categories 1 and 2) there were 76 (51.4\%) patients (mean age 56.1 \pm 9.6 ; ranging from 22 to 79 years). 35 (46.1\%) of those women had breast cancer, while 41 (53.9\%) women had benign lesions. In this 


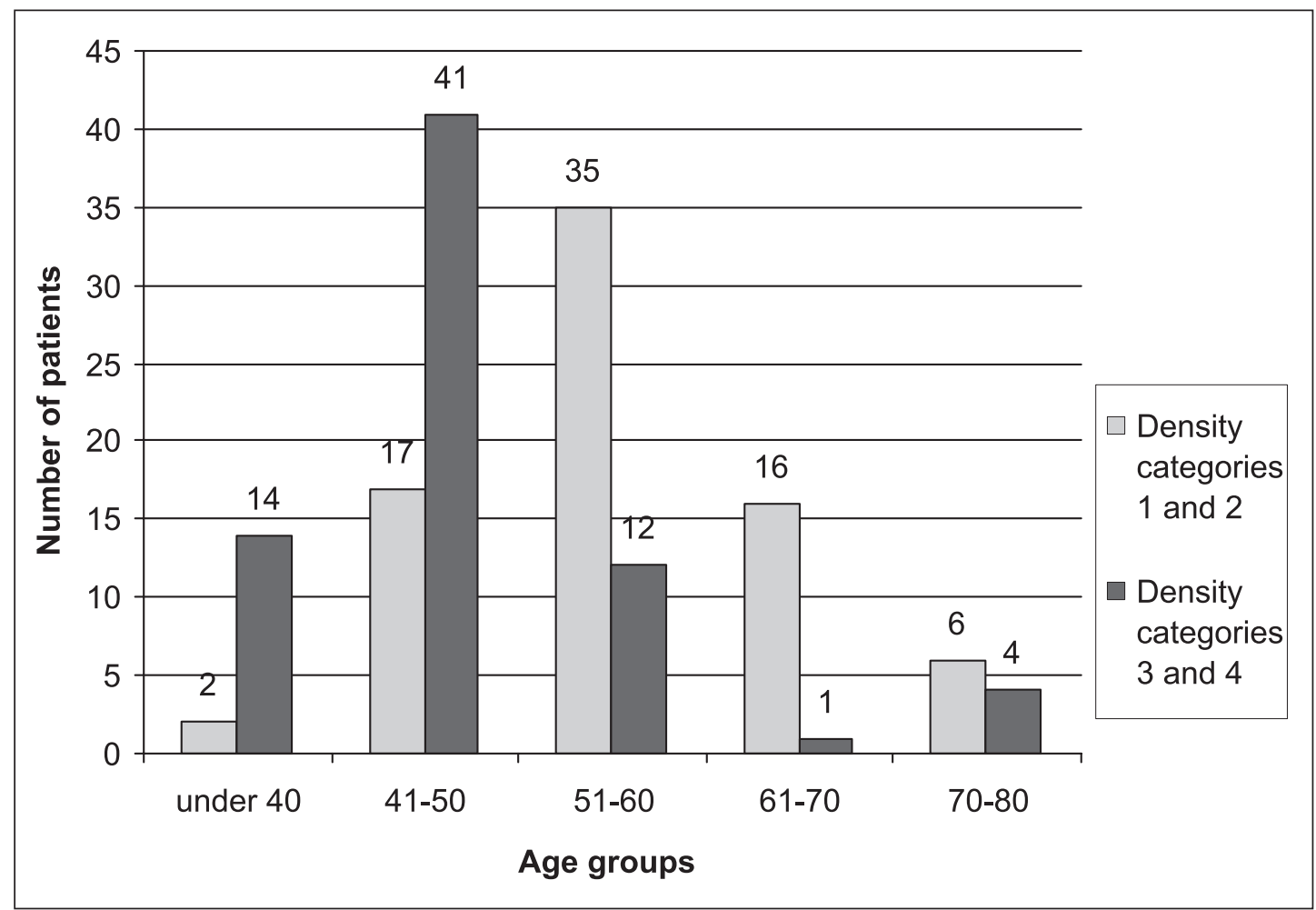

Figure 1 Distribution of breast density categories in different age groups

Table 1 The sensitivity and specificity of ultrasound and mammography in women with fatty breast (density categories 1 and 2)

\begin{tabular}{lll}
\hline \multirow{2}{*}{ Reliability } & \multicolumn{2}{l}{ Diagnostic procedures } \\
\cline { 2 - 3 } & Mammography $\left(95 \% \mathrm{Cl}^{*}\right)$ & Ultrasound $\left(95 \% \mathrm{Cl}^{*}\right)$ \\
\hline Sensitivity (\%) & $94.3(80-99)$ & $97.1(85-99)$ \\
Specificity (\%) & $68.3(51-81)$ & $82.9(67-92)$ \\
Positive predictive value (\%) & $71.7(56-84)$ & $82.9(67-92)$ \\
Negative predictive value (\%) & $93.3(77-99)$ & $97.1(85-99)$ \\
Positive likelihood ratio & $3(1.9-4.7)$ & $5.7(2.9-11.2)$ \\
Negative likelihood ratio & $0.08(0.02-0.32)$ & $0.03(0.004-0.23)$ \\
\hline
\end{tabular}

$\mathrm{Cl}^{*}=$ confidence interval

group, $75 \%$ of the women were older than 50 years while $25 \%$ women were younger than 50 years. The ultrasound sensitivity in this group was $2.8 \%$ higher than mammographic sensitivity, while the specificity of ultrasound was $14.6 \%$ higher than mammography (Table $1)$. In this group the difference in the sensi- tivities of the two imaging tests was not statistically significant $\mathrm{p}=1\left(\chi^{2}=0\right)$. Also, the difference in the specificities of the two imaging tests was not statistically significant $\mathrm{p}=0.11$ $\left(\chi^{2}=2.5\right)$ (Table 1).

In group B (women with dense breastdensity categories 3 and 4) there were 72 
Table 2 The sensitivity and specificity of ultrasound and mammography in women with dense breasts (density categories 3 and 4)

\begin{tabular}{lll}
\hline \multirow{2}{*}{ Reliability } & \multicolumn{2}{l}{ Diagnostic procedures } \\
\cline { 2 - 3 } & Mammography $\left(95 \% \mathrm{Cl}^{*}\right)$ & Ultrasound $\left(95 \% \mathrm{Cl}^{*}\right)$ \\
\hline Sensitivity (\%) & $53.6(33-72)$ & $85.7(67-95)$ \\
Specificity (\%) & $63.6(47-77)$ & $77.3(62-88)$ \\
Positive predictive value (\%) & $48.4(30-66)$ & $70.6(52-84)$ \\
Negative predictive value (\%) & $68.3(51-81)$ & $89.5(75-97)$ \\
Positive likelihood ratio & $1.5(0.9-2.5)$ & $3.8(2.1-6.6)$ \\
Negative likelihood ratio & $0.73(0.46-1.15)$ & $0.18(0.07-0.46)$ \\
\hline
\end{tabular}

$\mathrm{Cl}^{*}=$ confidence interval

(48.6\%) patients (mean age $47 \pm 10.1$; ranging from 19 to 77 years). 28 (38.9\%) of these women had breast cancer and $44(61.1 \%)$ women had benign lesions. $76.4 \%$ of the women in this group were younger than 50 years, but $23.6 \%$ women older than 50 years also had dense breasts.

The ultrasound sensitivity in the women with dense breasts was $32.1 \%$ higher than the mammographic sensitivity. In this group the ultrasound sensitivity was significantly higher than the mammographic sensitivity $\left.\left(\mathrm{p}=0.03 \chi^{2}=4.92\right)\right)$. The specificity of ultrasound was $13.7 \%$ higher than mammography but the difference in the specificities of the two imaging tests was not statistically significant $\left(\mathrm{p}=0.26, \chi^{2}=1.25\right)$ (Table 2$)$.

The regression of the probability of positive mammographic results on breast density in patients with cancers (sensitivity) was significant (likelihood ratio $\chi 2=15.99$, $\mathrm{p}=0.0001$ ) while the regression of the probability of a positive ultrasound result on breast density in cancers was not significant (likelihood ratio $\chi^{2}=1.96, p=0.161$ ). With the increase in breast density, the odds ratio for (the probability of - ex) a positive mammographic result was 0.25 (95\% CI, 0.110.58 ), and the odds ratio for (the probability of - ex) a positive ultrasound result was 0.52 (95\% CI, 0.19-1.37). These results indicate that a significant linear relationship exists between breast density and the logarithm odds of a positive mammographic result, while there is no linear relationship between breast density and the logarithm odds of a positive ultrasound result.

The regression of the probability of a negative mammographic result on breast density in patients without cancer (specificity) was not significant for mammography (likelihood ratio $\left.\chi^{2}=1.23, p=0.27\right)$. The regression of the probability of a negative ultrasound result on breast density in patients without cancer was also not significant (likelihood ratio $\chi^{2}=0.82$, $\mathrm{p}=0.36$ ). With the increase in breast density, the odds ratio for (the probability of - ex) a negative mammographic result was 0.75 (95\% CI, 0.45-1.25), and the odds ratio for (the probability of - ex) a negative ultrasound result was similar, 0.76 (95\% CI, 0.42-1.39). These results indicate that no significant linear relationship exists between breast density and the logarithm odds of a negative mammographic or ultrasound result.

\section{Discussion}

Women with breast disease symptoms or palpable findings on clinical examination are usually examined by mammography or breast ultrasound or both. The choice of the 
primary breast imaging in examining women with symptoms is partly based on age (2). One of the factors leading to false-negative findings on mammography is the effect of breast density $(6,10)$. Greater breast density is not only related to decreased sensitivity of mammograms because of its masking effect but it is also a major independent risk factor for breast cancer (11). A study of more than 200,000 women showed that the breast cancer risk is 5 times increased in the case of dense breasts compared to women with involutional changes (12). Higher breast density increases cancer risk in addition to the effects of other risk factors, and modifies the effects of body mass index and oral contraceptive use (13). Postmenopausal women with high breast density are at increased risk of breast cancer (14).

The mammographic appearance of the breast tissue varies depending on the tissue composition. Breasts with low density have a high proportion of fatty tissue, whereas breasts with high density have a high proportion of epithelial and connective tissue. In women with heterogenous and dense breasts, dense tissue obscures the radiological picture and makes the identification of cancers more difficult $(4,9,15,16)$.

The advantage of breast ultrasound compared to mammography increases with higher breast density and in young women, where the sensitivity of mammography is low. This is an important fact because more than half of the women younger than 50 years have heterogeneously dense or very dense glandular breast tissue, while one third of women older than 50 years also have dense breasts (17). Kim et al. (18) examined the prevalence of heterogeneous breasts and extremely dense breasts (density categories 3 and 4) in different age groups in Korean women and compared them with known results from western women. In Korean women, the frequency of dense mammogram was $88.1 \%$ (30-34 years old), 91.1\% (35-39),
$78.3 \%(40-44), 61.1 \%(45-49), 30.1 \%$ (50$54), 21.1 \%$ (55-59), and 7.0\% (60-64). Korean women in their 40s thus showed a higher frequency of dense mammograms, but this frequency decreased abruptly between the ages of 40 and 54 . In western women, there was little difference between 40 and 54 yearolds. It is obvious from these results that a very large percentage of women aged from 40 to 54 belong to density categories 3 and 4 which significantly reduces the sensitivity of mammography, which is used as the screening method after 40. In our study, 87.5\% women under 40 years had dense breasts; $70.7 \%$ women aged $41-50 ; 25.5 \%$ women aged 51-60 and even $18.5 \%$ women above 60 years. These results are similar to Kim's results (18), but in our study, we had more women above 60 with dense breast than Kim et al.

Several studies have shown that detection of breast cancer with mammography is limited in young women and in menopausal women with dense breast tissue $(12,19)$. Some studies compared the sensitivity of mammography and ultrasound in different density categories $(2,11,20,21,22,23)$.

Devolli-Disha et al. (2) in their study conducted on 546 patients with breast symptoms proved that for heterogenous and dense breasts, ultrasound is significantly more sensitive than mammography $(\mathrm{p}<0.01)$. In their study, mammographic sensitivity in women with predominantly fatty breasts was $82.2 \%$ while in the women with dense breasts it was only $23.7 \%$. The specificity of mammography for predominantly fatty breasts was $100 \%$, for heterogeneous breasts $63.5 \%$, while for extremely dense breasts it was only $16.3 \%$. The specificity of ultrasound in their study decreased from $100 \%$ in women with predominantly fatty breasts to $72.1 \%$ in women with extremely dense breasts.

Other studies, conducted on asimptomatic women, also proved the higher sensitivity of ultrasound than mammography 
in women with dense breasts. Leconte et al. (20) found in women with fatty breast $80 \%$ of cancers were diagnosed by mammography and $88 \%$ by ultrasound. In women with dense breasts the sensitivity of mammography was decreased to $56 \%$, while the sensitivity of ultrasound was still $88 \%$.

In $2002 \mathrm{Kolb}$ et al. (9) published results from a study that was conducted on 11,130 women. In their study, the sensitivity of mammography which was $98 \%$ in women with fatty breasts decreased to $48 \%$ in women with extremely dense breasts, while the sensitivity of ultrasound in that group was $75 \%$. Crystal et al. (4) in a study of 1517 asimptomatic women, proved the $100 \%$ sensitivity of ultrasound in women with dense breasts.

In contrast to previous studies, Barlow et al. (24) reported high mammographic sensitivity even in dense breasts. In their study, the mammographic sensitivity in women with almost entirely fat breasts was $86.3 \%$, in women with scattered fibroglandular densities $90.1 \%$, in women with heterogeneously dense breasts $82.9 \%$, and in women with extremely dense breasts the mammographic sensitivity was $81 \%$. The sensitivity difference of mammography between the different density groups was not high. In their study, the specificity of mammography, which was $92.5 \%$ in women with almost entirely fat breasts, decreased to $83.5 \%$ in women with extremely dense breasts.

In our study, the sensitivity of mammography was linearly associated with breast density while the sensitivity of ultrasound and the specificity of each test were not. The ultrasound sensitivity in the women with dense breasts was significantly higher than the mammographic sensitivity, while in women with fatty breasts the difference in the sensitivities of the two imaging tests was not statistically significant, which is in accordance with published results $(2,20,9)$. The sensitivity of mammography, which was $94.3 \%$ in women with fatty breasts, decreased to $53.6 \%$ in women with dense breasts, while the sensitivity of ultrasound decreased by $11.4 \%$ between these groups. The decrease in the sensitivity of mammography between women with fatty and dense breasts is in accordance with the results of some other studies $(2,9,20)$ but not in accordance with the results published by Barlow et al. (24), who found similar sensitivity of mammography in women with fatty breasts and in women with dense breasts. The decrease in the sensitivity of mammography was lower than in the study conducted by Devolli-Disha et al. (2), which was conducted on breast symptomatic women, as in our study. Our results are much more similar to the results of some other studies conducted on asymptomatic women $(9,20)$. In our study, the difference in the ultrasound specificity between groups was $5.6 \%$, and the difference in the mammography specificity between women with dense breasts and women with fatty breasts was only $4.7 \%$. This last result is only $4.2 \%$ lower than the results found by Barlow et al. (24).

\section{Conclusion}

Breast density has a significant influence on the sensitivity of mammography but not on specificity. This is very important because a certain percentage of women, not only under 40 but also those in their 40s, 50s and even 60s, have heterogenous and extremely dense breasts (breast density categories 3 and 4). In these women, the ultrasound is a more accurate imaging test than mammography, while in women with fatty breasts (breast density categories 1 and 2) these imaging tests are almost equally accurate in breast cancer diagnosis.

Conflict of interest: The authors declare that they have no conflict of interest. This study was not sponsored by any external organisation.

Authors' contributions: Conception and design: SM; Acquisition, analysis and interpretation of data: SM, MB, HF; Drafting the article: SM; Revising it critically for important intellectual content: SM. 


\section{References}

1. McPherson K, Steel M, Dixon J. Breast cancerepidemiology, risk factors, and genetic. Brit Med J. 2000;321:624-8.

2. Devolli-Disha E, Manxhuka-Kerliu S, Ymeri H, Arben K. Comparative accuracy of mammography and ultrasound in women with breast symptoms according to age and breast density. Bosnian journal of basic medical sciences. 2009;9(2):133-6.

3. Bassett LW, Kimme-Smith C. Breast sonography. AJR. 1991;156:449-55.

4. Crystal P, Strano SD, Shcharynski S. Using sonography to screen women with mammographically dense breasts. AJR Am J Roentgenol. 2004; 182:259-60.

5. Kerlikowske K, Grady D, Barclay J, Sickles EA, Ernster V. Effect of age, breast density, and family history on the sensitivity of first screening mammography. JAMA. 1996;276:33-8.

6. Oza AM, Boyd NF. Mammographic parenchymal patterns: a marker of breast cancer risk. Epidemiol Rev. 1993;15:196-208.

7. Boyd NF, Lockwood GA, Martin LJ, Knight JA, Jong RA, Fishell E, et al. Mammographic densities and risk of breast cancer among subjects with a family history of this disease. J Natl Cancer Inst. 1999;91:1404-8.

8. American College of Radiology. Illustrated Breast Imaging Reporting and Data System (BI-RADS) 3rd ed. Reston, Va: American College of Radiology, 1998.

9. Kolb TM, Lichy J, Newhouse JH. Comparison of the performance of screening mammography, physical examination, and breast US and evaluation of factors that influence them: an analysis of 27.825 patients evaluations. Radiology. 2002;225:165-75.

10. Saarenmaa I, Salminen T, Geiger U, Heikkinen $\mathrm{P}$, Hyvärinen S, Isola J, et al. The effect of age and density of the breast on the sensitivity of breast cancer diagnostic by mammography and ultrasonography. Breast Cancer Res Treat. 2001;67:117-23.

11. Pinsky RW, Helvie MA. Mammographic breast density: effect on imaging and breast cancer risk. J Natl Compr Canc Netw. 2010;8(10):1157-64.

12. Boyd NF, Guo H, Martin LJ, Sun L, Stone J, Fishell E, et al. Mammographic density and the risk and detection of breast cancer. N Engl J Med. 2007;356:227-336.

13. Wong CS, Lim GH, Gao F, Jakes RW, Offman J, Chia KS, Duffy SW. Mammographic den- sity and its interaction with other breast cancer risk factors in an Asian population. Br J Cancer. 2011;1;104(5):871-4.

14. Kerlikowske K, Cook AJ, Buist DS, Cummings SR, Vachon C, Vacek P, Miglioretti DL. Breast cancer risk by breast density, menopause, and postmenopausal hormone therapy use. 2010;20:28(24):3830-7.

15. Mandelson MT, Oestreicher N, Porter PL, White D, Finder CA, Taplin SH, White E. Breast density as a predictor of mammographic detection: comparison of interval- and screen-detected cancers. J Natl Cancer Inst. 2000;92:1081-108.

16. Olsen AH, Bihrmann K, Jensen MB, Vejborg I, Lynge $\mathrm{E}$. Breast density and outcome of mammography screening: a cohort study. Br J Cancer. 2009;7;100(7): 1205-8.

17. Stomper PC, D’Souza DJ, Di Notto PA, Arrendondo MA. Analysis of parenchymal density on mammograms in 1353 women 25-79 years old. AJR Am J Roentgenol. 1996;167:1261-5.

18. Kim SH, Kim MH, Oh KK. Analysis and Comparison of Breast Density according to on Mammogram between Korean and Western Women. J Korean Radiol Soc. 2000;42(6):1009-14.

19. Elmore JG, Armstrong K, Lehmann CD, Fletcher SW. Screening for breast cancer. JAMA. 2005;293:1245-56.

20. Leconte I, Feger C, Galant C, Berlière M, Berg BV, D'Hoore W, Maldaque B. Mammography and subsequent whole-breast sonographv of nonpalpable breast cancers. The importance of radiology breast density. AJR Am J Roentgenol. 2003;180:1675-9.

21. Kolb TM, Lichv J, Nevvhouse JH. Occult cancer in women with dense breasts Detection with screening US-diagnostic yield and tumor characteristics. Radiology. 1998;207:191-9.

22. Ciatto S, Rosselli del Turco M, Catarzi S, Morrone D. The contribution of ultrasonography to the differential diagnosis of breast cancer. Neoplasma. 1994;41:341-5.

23. Ma L, Fishell F, Wright B, Hanna W, Allan S, Boyd NF. Case-control study of factors associated with failure to detect breast cancer by mammography. J Natl Cancer Inst. 1992;84:781-5.

24. Barlow WE, Lehman CD, Zheng Y, Ballard-Barbash R, Yankaskas BC, Cutter GR et al. Performance of diagnostic mammography for women with signs or symptoms of breast cancer. J Natl Cancer Inst. 2002;7;94(15):1151-9. 